\title{
Exhibition
}

\section{Eyes that follow you around the room}

Since 1979, the industrial city of Linz in Austria has been the unlikely home of Ars Electronica, a centre for the development and dissemination of digital art and media. The centre has evolved to include an annual festival, a 'cyberarts' competition and 'The Futurelab', a media lab where interdisciplinary collaborators design and engineer new installations.

To celebrate Ars Electronica's 25th anniversary, New York City's American Museum of the Moving Image is hosting Digital AvantGarde, a series of exhibitions and screenings of projects that featured prominently in earlier competitions. Linz will have its own anniversary celebration during the first week of September.

The New York series, which runs until 18 July, includes John Gerrard's Portrait Diptych: Nadia (formerly called Networked Portrait), an interactive portrait that can be changed with the touch of a finger on a screen. The installation, shown here, consists of two liquid-crystal-display touchscreens, each presenting the computer-generated image of a particular individual.

By dragging a finger across an eye or the

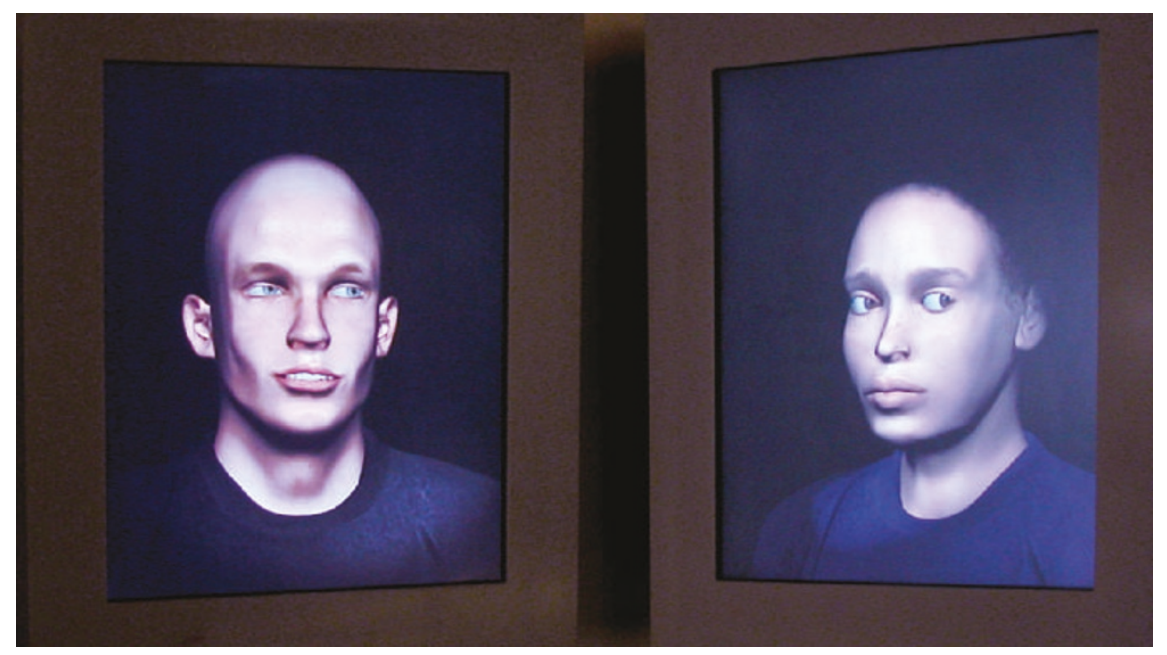

corner of the mouth in one of the images, the viewer can impart some emotion to the otherwise expressionless visage. When the screens are turned toward each other, the other face responds by subtly changing its own expression. The two faces then continue to respond to each other.
The portrait is seen as one of the last bastions of permanence at a time when images can be erased or modified at will. But even the portrait, it seems, can be digitized and updated continually — for example, to reflect one's mood.

Alan Packer planet is significant, perhaps being uniquely situated to foster both complex life and scientific discovery. Gonzalez and Richards believe that intelligence may be a rare phenomenon in our galaxy. They make their case, in part, by drawing on arguments others have given before, such as Peter D. Ward and Donald Brownlee in Rare Earth (Springer, 2000). But they go far beyond estimating the prevalence of extraterrestrial life. As they summarize: "The myriad conditions that make a region habitable are also the ones that make the best overall places for discovering the universe in its smallest and largest expressions. This is the central argument, the central wonder of this book."

Drawing on a framework for inferring design proposed by philosopher and mathematician William Dembski in The Design Inference (Cambridge University Press, 1998), Gonzalez and Richards argue that the correlation between the conditions that make habitability possible and those that make it possible to learn about the Universe is so exquisite and improbable as to suggest intelligent design.

For example, by raising the tides the Moon helps move nutrients from the land to the ocean, fostering life in the intertidal zone. If the Moon were farther from the Earth, the authors argue, it would need to be much larger to have the same effect on the tides; if it were closer, it might well be less spherical, causing other problems. So, Gonzalez and Richards argue, the Moon is located at a distance from Earth that is very conducive to life. It is also at an optimal distance for scientific discovery (or measurability), they contend, as the disk of the Moon provides a perfect eclipse of the Sun. They argue that without such perfect solar eclipses, humans would have been deprived of important information about the Sun's chromosphere.

A single such instance linking habitability and measurability might be dismissed as a coincidence, but Gonzalez and Richards claim that the correlation across many phenomena cannot be explained by chance. By including astronomical and geological phenomena in their search for evidence of purpose in the cosmos, The Privileged Planet expands the discussion of intelligent design far beyond its usual contemporary focus on the complexity of biological systems and the fundamental physical constants.

Ultimately, however, the authors are in a poor position to argue that Earth is optimally located for both habitability and measurability. They try to establish habitability requirements by comparing Earth with other locations in the galaxy. Unfortunately, we lack the data required for a well-reasoned comparison. If we had many examples of planets that do and do not bear life, and an explanation for why the conditions on some planets led to life while those on others did not, we might be able to establish an accurate metric of habitability. Until then, we are forced to extrapolate measures of habitability from a sample of one inhabited planet.

Regrettably, anyjudgements of optimality may be biased by the local conditions and historical contingencies through which our life and our science have evolved, rather than accurately reflecting the range of possible preconditions for habitability and measurability. Potential readers of The Privileged Planet would do well first to familiarize themselves with the biases that can result from this kind of selective sampling. A good primer is Nick Bostrom's Anthropic Bias (Routledge, 2002).

Caution seems especially in order given that the authors have intentionally limited themselves to our knowledge of the Universe gained through selected observational sciences, such as comparative planetary geology, solar physics and astronomy, rather than including more laboratory-based sciences. Similarly, although the authors attempted to avoid cherry picking instances of measurability that support their position by focusing on important observations in these fields, the vagueness of such a criterion makes their selection rather subjective.

So far, Earth is the only planet we know that has the privilege of bearing life that searches for signs of other intelligence whether in the form of other technological beings transmitting evidence of their existence or through patterns indicating underlying design. It may be some time, however, before we can accurately judge whether our blue dot is - as planets go - commonplace, unique or somewhere in between.

Douglas A. Vakoch is at the SETI Institute,

2035 Landings Drive, Mountain View,

California 94043, USA. 